\title{
Nur begrenzt positive Wirkungen
}

\author{
Die Beschäftigung im Umweltschutz ist eine wichtige Option in der akfuellen \\ politischen Debatte, verspricht sie doch zwei zentralen Krisen unserer \\ Gesellschaft entgegenzuwirken: der Arbeitslosigkeit und der Zerstörung \\ natürlicher Ressourcen. Vor allem in den neuen Bundesländern hat die öffent- \\ liche Beschäftigungsförderung im Umweltbereich eine wesentliche Bedeutung \\ gespielt. Kann sie die Hoffnung auf eine Funktion als Brücke in den ersten \\ Arbeitsmarkt erfüllen?
}

D

Von Ulrich Petschow, Regine Stein und Eckart Hildebrandt

üpfung von Umweltschutz und Erwerbsarbeit gilt als eine der großen Chancen, den gegenwärtig herrschenden sozio-ökonomischen und ökologischen Krisen unserer Gesellschaft entgegenzuwirken: der Massen- und Dauerarbeitslosigkeit auf der einen Seite und der zunehmenden Zerstörung natürlicher Ressourcen auf der anderen Seite. Aufgrund dieses doppelten Wirkungspotentials ist die Beschäftigung im Umweltschutz zu einer der wichtigsten Optionen in der aktuellen politischen Debatte geworden. Zugleich haben sich die Vorstellungen über die Rolle eines zweiten, staatlich geförderten Arbeitsmarktes stabilisiert. Vor diesem Hintergrund gewinnen die Erfahrungen in den neuen Bundesländern an Bedeutung, da dort die öffentliche Beschäftigungsförderung im Umweltbereich eine wesentliche Rolle gespielt hat (1).

\section{Beschäftigungsförderung in den neuen Bundesländern}

In Ostdeutschland waren 1991 insgesamt knapp 200.000 Menschen in Arbeitsbeschaffungsmaßnahmen (ABM) beschäftigt, das Verhältnis zur Arbeitslosenzahl betrug damit 1:4,8. 1992 wurde dann auf dem Höhepunkt der ABM-Förderung in den neuen Bundesländern mit rund 387.000 ABM-Beschäftigten ein Verhältnis von 1:3 erreicht. 1996 - nachdem 1993 der pauschalierte Lohnkostenzuschuß nach $\S 249$ h des Arbeitsförderungsgesetzes (LKZ) als weitere Maßnahmeart hinzugekommen war - lag dieses Verhältnis mit 277.000 Beschäftigten in $\mathrm{ABM}$ oder LKZ immer noch bei 1:4,2. Im Vergleich gab es auf dem - quantitativen - Höhepunkt der öffentlichen Beschäftigungsförderung in Westdeutschland 1988 insgesamt knapp 115.000
Beschäftigte in ABM, was einem Verhältnis von ABM-Beschäftigten zu Arbeitslosen von 1:19 entsprach. 1993 lag dieses Verhältnis (bei gut 50.000 ABM-Beschäftigten) in Westdeutschland dann bei 1:45 (2).

Die Motivation zur Teilnahme an einer Maßnahme resultiert in der Regel nicht aus bestimmten Maßnahmeinhalten - wie etwa Umweltaspekten -, sondern vor allem aus der Perspektive, nach Abschluß der Maßnahme eine Arbeitsstelle zu bekommen. Dies trifft vor allem dann zu, wenn die Maßnahme selbst in keinem Zusammenhang mit der jeweiligen individuellen Qualifikation steht.

In den dominanten LKZ-Maßnahmebereichen „Wirtschaftsnahe Infrastruktur" und „,Forstwirtschaft/Landschaftspflege/Naturschutz" entfallen 85 Prozent der Tätigkeiten auf eher einfache bzw. mittlere Arbeitertätigkeiten, für die eine Anlernphase und eher geringe formale Qualifikationen ausreichen (3). Die Arbeiten bestehen z. B. aus Beräumung und Abriß oder dem Rückschnitt von Grabenwucherungen. Spezialistentätigkeiten sind nur in Ausnahmefällen zu erbringen, die Arbeiten bedürfen im allgemeinen nur weniger Fachwissensträger zur Anleitung, was zugleich den Betreuungsaufwand gering hält. Angesichts der Tatsache, daß zum Befragungszeitpunkt über 60 Prozent der LKZGeförderten in diesen beiden Bereichen beschäftigt waren, ergibt sich aus der Gegenüberstellung mit den Qualifikationsstrukturen weiterhin hohe Facharbeiter/innen-Anteile -, daß viele Maßnahmeteilnehmer/innen nicht ihrer formalen Qualifikation gemäß eingesetzt wurden.

Zudem haben (Weiter-)Qualifizierungen oft einen geringeren Anteil als die zulässigen 20 Prozent an der Arbeitszeit und sind eng an dem
Anforderungsprofil der konkreten Maßnahme ausgerichtet, so daß sie häufig nicht $\mathrm{zu}$ einer Qualifikationsanhebung führen, die für den ersten Arbeitsmarkt relevant ist. Ebensowenig sind sie auf Lernprozesse im Umweltschutz ausgerichtet.

Über Anschlußperspektiven werden sehr unterschiedliche Angaben gemacht. Trotz der Problematik der Vergleichbarkeit sind die Quoten mit bis zu 20 Prozent des Übergangs in den ersten Arbeitsmarkt eher gering. In den neuen Bundesländern hatten die (Groß-)Maßnahmen im Umweltbereich (LKZ) nur begrenzte positive Wirkungen, sie schnitten schlechter ab als Maßnahmen in anderen Bereichen. (FN: Schüssler, R., 1997: Evaluierung der Arbeitsförderung Ost gemäß 249h. Nicht autorisierte Fassung.)

Mit Blick auf das Ausgründungspotential der „Gesellschaften zur Arbeitsförderung, Beschäftigung und Strukturentwicklung“ (ABS) gingen die ersten Einschätzungen von bis zu zehn Prozent der Beschäftigten aus. Die Hoffnungen bezogen sich aufgrund der Marktnähe vor allem auch auf den Umweltbereich. Bereits 1993 wurde deutlich, daß die Erwartungen bei weitem überzogen waren. Knuth stellte fest, daß bis dahin aus ABS die Schaffung von insgesamt 2.002 Arbeitsplätzen durch Existenz- und Ausgründungen erreicht werden konnte, was nach seiner Berechnung einer Quote von 1,8 Prozent entsprach (4). Die Ausgründungen haben vor allem in den Bereichen ,Handwerk" und ,Industrielle Sanierung" stattgefunden. „Umwelt" ist hier insofern relevant, als durch die Umweltsanierung Nachfrage in diesen konventionellen Märkten induziert wird. Ausgründungen im Umweltbereich im engeren Sinne spielen demgegenüber eine eher untergeordnete Rolle.

\section{Perspektiven}

Aus den Ergebnissen muß gefolgert werden, daß insbesondere die Großmaßnahmen, wie sie vorrangig in den ABS-Gesellschaften durchgeführt wurden, über die soziale Abfederungsfunktion und die Basis für die Strukturentwicklung hinaus nur sehr begrenzt positive Wirkungen entfalten konnten. Insofern ergibt sich in der Perspektive die Notwendigkeit, die Beschäftigungsförderung deutlich mehr auf kleinteiligere Projekte zu fokussieren, wobei sich erneut die Frage nach relevanten Tätigkeitsbereichen stellt.

Die bisher vorrangig bedeutsamen Beschäftigungsbereiche im nachsorgenden Umweltschutz erweisen sich in der Perspektive als nur 
begrenzt ausbau- und entwicklungsfähig. Ein Übergang in die unmittelbare Umweltbeschäftigung im ersten Arbeitsmarkt wird nur in Ausnahmefällen gelingen können. Entscheidend für die öffentliche Beschäftigungsförderung im Umweltbereich erscheint es, in Zukunft verstärkt an den herkömmlichen Tätigkeitsfeldern anzusetzen und dort ökologische Potentiale zu identifizieren und zu erschließen. Sie könnte einen explorativen Charakter haben, indem ökologische Nischen ggf. arbeitsintensiv getestet und ausgelotet werden. Das sagt allerdings noch nichts dariber aus, inwiefern solche Tätigkeitsfelder in der Folge aus der öffentlichen Beschäftigungsförderung herauswachsen und sich einen eigenen Markt schaffen könnten. Ein Beispiel aus der Abfallwirtschaft zeigt die mögliche Dynamik.

Die Abfallwirtschaft und dabei vorrangig die Recyclingwirtschaft waren und sind weiterhin ein wesentliches Betätigungsfeld des zweiten Arbeitsmarktes. So konnten z. B. gerade im Bereich Elektronikschrott oder auch der „weißen Ware“ durch die Subventionierung des Faktors Arbeit Ansätze der Marktfähigkeit entwickelt werden. Diese Maßnahmen sind in der Regel stark manuell und insofern ,vorindustriell“ ausgerichtet. Wenn die Bereiche von staatlicher Regulierung erfaßt und Umweltstandards umgesetzt werden, werden die Märkte für die öffentliche Beschäftigungsförderung in Frage gestellt.

Dies kann an der Aufarbeitung der ,weißen Ware" nachgewiesen werden. In diesem Bereich waren über lange Zeit $z$. B. die sozialen Betriebe tätig. Mittlerweile ist das Feld von den großen Unternehmen der Entsorgungswirtschaft als Markt entdeckt worden. Damit verändern sich die Marktstrukturen deutlich: Die Sammlung der entsprechenden Geräte erfolgt nunmehr großflächig, die Demontagebetriebe werden zentralisiert und machen die Demontage zu einem industriellen Prozeß. Dies führt zu einem deutlichen Rïckgang der Arbeitsintensität und somit zu einem ,normalen“ Tätigkeitsbereich.

In diesem Sinne sind eine Reihe von umweltorientierten Maßnahmen gegenwärtig beschäftigungsintensiv, weil sie auf vorindustriellem Niveau betrieben werden. Sobald diese Tätigkeitsfelder Marktverhältnissen unterworfen werden, werden sie stärker kostenorientiert ausgerichtet und die Rationalisierungsmöglichkeiten genutzt. Damit unterliegen umweltorientierte Maßnahmen im Hinblick auf die
Beschäftigungswirkung einer gewissen Dynamik. So würde z. B. die Beschäftigungsintensität der großen Sanierungsmaßnahmen in den neuen Bundesländern bei marktlicher Leistungserbringung nicht aufrechtzuerhalten sein. Damit verlieren die Maßnahmen im Umweltschutz aber zu einem Teil ihre arbeitsmarktpolitische Wirkung. Doch ist gerade im Abfallbereich auch darauf hinzuweisen, daß die Arbeitsqualität entsprechender Maßnahmen vielfach als - u. a. gesundheitlich - problematisch einzuschätzen war, etwa das Beispiel der Abfallsortierung.

Nach den vorliegenden Erfahrungen aus der aktiven Arbeitsmarktpolitik sind auch weiterhin die Erwartungen nicht zu hoch zu hängen. Aus arbeitsmarktpolitischen Gründen bleibt es unabdingbar, Maßnahmen wie bisher zur Entlastung des Arbeitsmarktes durchzufuihren. Dies sollte jedoch auf eine differenziertere Art und Weise geschehen: In der Summe bedeuten die Problemlagen der neuen Bundesländer, daß die aktive Arbeitsmarktpolitik in einen engen Zusammenhang mit den regionalen Entwicklungskonzepten und der Entwicklung der Unternehmen des ersten Arbeitsmarktes zu bringen ist. Sie sollte dabei an drei wesentlichen Säulen ansetzen:

- Weiterführung bestehender Projekte der Beschäftigungsförderung,

- Förderung von Projekten mit innovativem Charakter und ggf. Ausgründungspotentialen,

- Unterstïtzung und Aufbau von Maßnahmen, die die lokale Subsistenz und Kommunarität entwickeln.

Für die Projektausrichtung bedeuten die zweite und dritte Säule, daß die breit angelegten, inhaltlich weitgehend gleichgerichteten Maßnahmen zu ergänzen sind durch spezialisiertere Maßnahmeninhalte, die an den regional- und qualifikationsspezifischen Bedingungen anknüpfen. Insbesondere für die zweite Säule ist es erforderlich, qualifikationsorientierte Zielgruppen zu bilden. Diese Projekte sollten Experimentier- und Testfelder für (noch) nicht im Markt befindliche ökologische Produkte und Dienstleistungen bieten. Eine Anbindung an den ersten Arbeitsmarkt, z. B. an regionale Unternehmen, wäre wünschenswert. Neben einer notwendigen Spezialisierung der Maßnahmeträger müssen darüber hinaus die entsprechenden zeitlichen und personellen Ressourcen zur Verfügung gestellt werden, $d$. h. die derzeitige Kurzatmigkeit der Arbeitsmarktpolitik muß - hin zu einer angemessenen Dauer von Maßnahmen - verändert werden sowie die Zuweisungspraxis der Arbeitsämter. Somit ist der vielfach gestellten Forderung nach verbesserter Koordinierung und Vernetzung der verschiedenen arbeitsmarktpolitischen Akteure erneut Nachdruck zu verleihen.

Im Sinne einer ökologischen Ausrichtung der Beschäftigungsförderung im Umweltbereich ergibt sich insbesondere für die zweite und dritte Säule, daß die Tätigkeiten selbst und auch die Qualifizierung nicht spezifisch ökologischer Art sein müssen, sondern vielmehr Ökologie als ein Aspekt unter anderen zu betrachten ist, der im Idealfall aber an jeder Stelle mitgedacht wird. Abschließend ist aber darauf hinzuweisen, daß mit der sich verfestigenden Arbeitslosigkeit mehr und mehr solche Aspekte wichtig werden, die weniger an den klassischen Arbeitsplätzen/Berufen orientiert sind, sondern vielmehr auf die neuen Unübersichtlichkeiten und insofern auf ein erweitertes Spektrum von Beschäftigung ausgerichtet sind. Generell werden in Zukunft Aspekte wie Eigenbeschäftigung an Gewicht gewinnen, die in der Lage sind, stabilisierende Funktionen in den Regionen wahrzunehmen und die Trennung zwischen Arbeitswelt und Lebenswelt $\mathrm{zu}$ reduzieren. Die öffentliche Beschäftigungsförderung sollte diesen veränderten Bedingungen auch vor dem Hintergrund der Nachhaltigkeitsdiskussion in stärkerem Maße Rechnung tragen.

\section{Anmerkungen}

(1) Der Beitrag basiert auf der vom IÖW 1997 durchgeführten Studie „Arbeitsqualität und Umwelteffektivität in der öffentlichen Beschäftigungsförderung" im Auftrag der Hans-Böckler-Stiftung.

(2) Sell, S.: Strukturwandel der Arbeitsbeschaffungsmaßnahmen; in: Zeitschrift für Sozialreform 9/1994, S. 807.

(3) Stark, B.: C. Wolfinger, Lohnkostenzuschüsse nach $\S$ 249h AFG - Erste Ergebnisse einer reprösentativen schriftlichen Befragung von Maßnahmeträgern Ende 1994/Anfang 1995; IABwerkstattbericht Nr. 11/1995, S. 26.

(4) Knuth, M.: Drehscheiben im Strukturwandel, Berlin 1996, S. 307.

Die Autorlnnen
Dipl. Volksw. Ulrich Petschow und Dipl.-Math. Regine
Stein sind wissenschaffliche Mitarbeiter am IÖW
Berlin, Dr. Eckart Hildebrandt ist wissenschaftlicher
Mitarbeiter am Wissenschaftszentrum Berlin
Kontakt: IÖW gGmbH, Giesebrechtstr. 13,
10629 Berlin, Tel. $(030) 884594$ - 0, Fax (030)
8825439


(c) 20I0 Authors; licensee IÖW and oekom verlag. This is an article distributed under the terms of the Creative Commons Attribution Non-Commercial No Derivates License (http://creativecommons.org/licenses/by-nc-nd/3.o/), which permits unrestricted use, distribution, and reproduction in any medium, provided the original work is properly cited. 